\author{
Paulo Eigi Miyagi \\ pemiyagi@usp.br \\ Editor-in-Chief \\ Newton Maruyama \\ maruyama@usp.br \\ JBSMSE - Electronic peer review system \\ manager
}

\section{The Mechanical Sciences and Engineering Research at the JBSMSE: a Snapshot of the Last Four Years}

This text is a snapshot of the last four years of the Journal of the Brazilian Society of Mechanical Sciences and Engineering (JBSMSE). The last four years is related to the period of the current editorial team which is coming to an end. At first, we present the evolution of the paper manuscript submission and peer review system towards an online electronic system, the SciELO system. This resulted on a much more reliable and efficient solution. After, a brief summary of the current peer review process is presented together with statistics of paper manuscripts submitted and approved for publication in each section. A qualitatively analysis of time duration for a peer review process is realized while trying to identify drawbacks and possible improvements. An analysis of the paper download requests via the SciELO system, during the past 4 years, is presented. Some of the most downloaded papers are listed together with their statistics. It is claimed here that number of downloads could be a possible Scientometrics index. At the end, we draw some conclusions on the current importance of the JBSMSE.

\section{Introduction}

This text is a snapshot of the last four years of the Journal of the Brazilian Society of Mechanical Sciences and Engineering (JBSMSE). As the period of the current Editorial Board is coming to an end, we would like here to briefly summarize the current developments that have been reached by this team.

At first, we present how the paper manuscript submission and peer review system evolved from an e-mail based system workflow, where database was managed on spreadsheet files, to an online electronic system, the SciELO system. The SciELO allowed us to have a complete solution for paper manuscript submission peer review system workflow together with an electronic online publishing system. This resulted on a much more reliable and efficient solution for all involved: editors, authors and reviewers. After, a brief summary of the current peer review process is presented together with statistics on number of paper manuscripts submitted and on number of paper manuscripts approved for publication in each section. Then, the current reviewing status in each section, i.e., a qualitatively analysis of time duration for a peer review process, is analyzed while trying to identify drawbacks and possible improvements. Later, an analysis of the paper download requests via the SciELO system, during the past 4 years, is presented. Some of the most downloaded papers are listed together with their statistics. It is claimed here that number of downloads could be a possible Scientometrics index. This proposal can be of course very controversial and must be considered carefully. At the end, we draw some conclusions on the current importance of the JBSMSE.

\section{Paper Submissions}

During the period of January 2006 to November of 2009, around 581 paper manuscripts were submitted (see Table 1). These submissions included cases that later, for some reason, were cancelled, and also, there were cases of submissions whose records were duplicated. However, we have noted that around 95\% of paper submissions were forwarded to the peer review process by one of the Associate Editors.

The column identified with "e-mail system", in Table 1 , is related to the period when paper manuscript submissions were realized by sending e-mail messages directly to the Editor-in-Chief. The whole workflow was realized by e-mail and the database was managed on spreadsheet files. The Editor-In-Chief was responsible for selecting the appropriate Associate Editor that would conduct the peer review process. This decision was based on the following aspects: author's suggestions, a preliminary analysis of the text, list of keywords and possible conflict of interests. This form of submission of papers was conducted until September 2006.

The e-mail based peer review system was, of course, very cumbersome and error prone. Therefore, it was decided to introduce an online electronic peer review system. The Open Journal Systems (OJS) Willinsky (2005) was our choice. The OJS is a journal management and publishing system that has been developed by the Public Knowledge Project and released under the GNU General Public License. OJS assists with the whole editorial management workflow from paper submission, multiple rounds of peer review to online publication and indexing. Due to a lot of restrictions, only the paper submission and peer review steps were utilized. All other steps were done manually. The research field for the peer review process was selected directly by authors. The Editor-in-Chief would verify for possible conflict of interests with the Associate Editor, and, if applicable he would choose another Associate Editor. When files for a journal issue were ready, they were sent for printing and to the SciELO (Scientific Electronic Library Online) for online publishing. The SciELO is a project under a partnership of the FAPESP (Research Support Foundation of the State of Sao Paulo), the BIREME (Latin American and Caribbean Center on Health Sciences), and many Brazilian and international institutions related to scientific communication and editors. The SciELO has also the support of the CNPq (National Council for Scientific and Technological Development of the Brazilian Ministry of Science and Technology). At the SciELO system, users can access JBSMSE papers on-line or download the corresponding PDF file. Our OJS was installed in a server named Proteus that is localized in one of 
the research labs of the Department of Mechatronics and Mechanical Systems Engineering at the EPUSP (Escola Politécnica da Universidade de São Paulo). This form of submission of papers was conducted until May 2009. The column identified with "Proteus system", in Table 1, summarizes the number of paper manuscripts submitted in each area.

During 2008, the managers of the SciELO system offered us the possibility of using the system for our workflow. The SciELO system is a parallel development version of the OJS system with minor modifications. By the beginning of 2009, user records at Proteus were transferred to the SciELO system. Unfortunately, the whole database could not be transferred. Since $15^{\text {th }}$ of May of 2009 only paper submissions through the SciELO system is being accepted. The workflow is the same as in the Proteus system. The SciELO system is used for paper submission, peer review and online publishing. The column identified as "SciELO system", in Table 1, summarizes the number of paper manuscript submissions in each area.

At this point in time, the Proteus system and the SciELO system are both active. The peer review process of all papers submitted prior to $15^{\text {th }}$ of May of 2009 must be followed at the Proteus system.
Table 1 presents the distribution of number of paper manuscripts submitted to each area. According to Table 1, the areas might be classified into 3 groups: (a) "high demand" that involves the areas of Manufacturing process; Dynamics, Vibration and Acoustics; and Fluid Mechanics, (b) "medium demand" that includes the areas of Thermal Sciences; Solid Mechanics; and Mechatronics and Robotics, and (c) "low demand" that involves the areas of Aerospace Engineering; Bioengineering; Nonlinear Phenomena, Product Engineering; Refrigeration, Heating, Ventilation and Air Conditioning; Combustion and Environmental Engineering; and Rheology and non-Newtonian Fluid Mechanics.

\section{The Peer Review Process}

Table 2 summarizes the number of paper manuscripts that were accepted for publication in each topic area after the peer review process conducted by one of the Associate Editors. For a paper manuscript to be accepted for publication it must go through two main steps. First, the Associate Editor sends the paper manuscript to at least two reviewers. Second, the Associate Editor must assess these reviews.

Table 1 - Manuscripts submitted by topic area.

\begin{tabular}{ccccc}
\hline Area & e-mail system & Proteus system & SciELO system & --Total-- \\
\hline Aerospace Engineering & 8 & 14 & 4 & 26 \\
Bioengineering & 3 & 16 & 4 & 23 \\
Combustion and Environmental Engineering & 1 & 12 & 7 & 20 \\
Dynamics, Vibration and Acoustics & 13 & 53 & 16 & 82 \\
Fluid Mechanics & 8 & 55 & 10 & 73 \\
Manufacturing Process & 15 & 87 & 13 & 115 \\
Mechatronics and Robotics & 4 & 28 & 8 & 40 \\
Nonlinear Phenomena & 7 & 10 & 1 & 18 \\
Offshore and Petroleum Engineering & 3 & 13 & 3 & 19 \\
Product Engineering & 2 & 15 & 4 & 21 \\
Refrigeration, Heating, Ventilation and Air Conditioning & 1 & 14 & 6 & 21 \\
Rheology and non-Newtonian Fluid Mechanics & 12 & 2 & 0 & 14 \\
Solid Mechanics & 9 & 33 & 9 & 51 \\
Thermal Sciences & 11 & 36 & & \\
Total & & & & 58 \\
\hline
\end{tabular}

Table 2 - Manuscripts approved for publication in each topic area.

\begin{tabular}{ccccc}
\hline Area & e-mail system & Proteus system & SciELO system & --Total-- \\
\hline Aerospace Engineering & 2 & 1 & 0 & 3 \\
Bioengineering & 2 & 2 & 0 & 4 \\
Combustion and Environmental Engineering & 1 & 8 & 0 & 9 \\
Dynamics, Vibration and Acoustics & 3 & 10 & 0 & 13 \\
Fluid Mechanics & 2 & 14 & 2 & 18 \\
Manufacturing Process & 5 & 24 & 1 & 30 \\
Mechatronics and Robotics & 0 & 6 & 2 & 8 \\
Nonlinear phenomena & 5 & 1 & 0 & 6 \\
Offshore and Petroleum Engineering & 2 & 1 & 0 & 3 \\
Product Engineering & 1 & 1 & 0 & 2 \\
Refrigeration, Heating, Ventilation and Air Conditioning & 0 & 1 & 0 & 2 \\
Rheology and non-Newtonian fluid mechanics & 3 & 1 & 0 & 4 \\
Solid mechanics & 4 & 7 & 0 & 11 \\
Thermal sciences & 4 & 2 & & $\mathbf{1 2 5}$ \\
\hline Total & & &
\end{tabular}


After this assessment, the Associate Editor attributes one of the following statuses to the manuscript: "Accept Submission", "Revisions Required", "Resubmit for review" and "Decline Submission". The "Resubmit for review" status indicates that a major review is required after resubmission for another peer review step.

We remind you that the Associate Editors have been chosen by nominations submitted by each Technical Committee of the ABCM. The final decision takes into account academic qualifications and also regional distribution. The academic qualifications of the editorial team are emphasized here by a sample of their publications: Bastos-Netto et al. (1997), Cunha \& Abade (2007), Caurin et al. (2001), Diniz \& Micaroni (2007), Maruyama \& Souza (2007), Morooka et al. (2001), Naccache et al. (2007), Rade et al. (2006), Savi et al. (2009), Zouain et al. (2006). Beyond qualifications, the work of Associate Editors involves appointment of reviewers, monitoring of workflow processes, assessment of reviews and decision about acceptance or rejection of paper manuscripts. The success of an Associate Editor work is dependent on strong commitment of the whole academic community, especially reviewers. These colleagues have voluntarily assumed this task in order to contribute for the development of the Mechanical Sciences and Engineering field, and we must acknowledge their efforts.

\section{Reviewing status per section}

The following facts highlight some aspects of the peer review process in each section.

- Aerospace Engineering - The topic area is classified, according to Table 1, as "low demand", but consistent with the number of Graduate courses of this field in Brazil. The reviewing process has usually required more time than the average of the JBSMSE.

- Bioengineering - The topic area is classified, according to Table 1 , as "low demand", but consistent with the number of Graduate courses of this field in Brazil. The reviewing process has usually required more time than the JBSMSE average.

- Combustion and Environmental Engineering - The topic area is classified, according to Table 1, as "low demand", but consistent with the number of Graduate courses in this field. Usually, the reviewing process is on schedule, which indicates the commitment of both reviewers and the Associate Editor, Professor Demetrio Bastos Neto.

- Dynamics, Vibration and Acoustics - The topic area is classified as "high demand" and, on average, the reviewing process is on schedule, but there is a relatively large time duration variance, i.e., indicating that reviewers commitment should increase in order to meet the deadlines and support the Associate Editor, Professor Domingos A. Rade.

- Fluid Mechanics - The topic area is classified as "high demand" and, on average, the peer review process is on schedule. The large number of paper manuscripts that are submitted to this area indicates that both reviewers and the Associate Editor, Professor Francisco R. Cunha, are working with great commitment.

- Manufacturing Process - The topic area is classified, according to Table 1, as "high demand". In fact, this field has the largest number of submissions. The average duration time of the peer review process meets the deadline. Considering the relatively large number of submitted paper manuscripts, it is an important indication of the large number of researchers and their efforts to the development of the field. In this topic area, we must specially acknowledge the excellent work of the Associate Editor, Professor Anselmo Diniz.
- Mechatronics and Robotics - The topic area is classified, according to Table 1, as "medium demand", with growing numbers. In general, the average time spent for the peer review process is on schedule, but there are few cases where deadlines have not met. The community must be conscious that reviewing processes must be on schedule in order to support the effort that has been carried out by the Associate Editor, Professor Glauco A. P. Caurin.

- Non-linear Phenomena - The topic area is classified, according to Table 1, as "low demand". Usually, the peer review process is on schedule. Both community and the Associate Editor, Professor Marcelo Savi, must be acknowledged.

- Offshore and Petroleum Engineering - The topic area is classified, according to Table 1, as "low demand". The peer review process has required a little more time than expected, the reduced number of researchers in this field might have influenced this result.

- $\quad$ Product Engineering - The topic area is classified, according to Table 1, as "low demand", which is expected due to the reduced number of Graduate courses in this field. Peer review processes have required more time than expected and in some cases much more time than what is considered acceptable. It is essential for the development of the Product engineering community that reviewers support the work of the Associate Editor.

- Refrigeration, Heating, Ventilation and Air Conditioning - The topic area is classified as "low demand". The peer review process has required more time than expected. Therefore we would like to see an increase of the community commitment in order to reduce the average duration time of the peer review process.

- Rheology and non-Newtonian Fluid Mechanics - The topic area is classified as "low demand". In general, the peer review process is on schedule. It is possible to note that even though the community of this field is very small they are working for the efficiency of the JBSMSE workflow. We must especially acknowledge the Associate Editor, Professor Monica Naccache, for the excellent work.

- Solid Mechanics - The topic area is classified, according to Table 1, as "medium demand", but with submissions growing consistently. In general, the peer review process is on schedule, but there are cases where deadlines were not met. It is important to increase the community commitment to complete the evaluation on schedule, and to support the effort that has been carried out by the Associate Editor, Professor Nestor Zouain.

- Thermal Sciences - The topic area is classified, according to Table 1, as "medium demand", but number of submissions is growing consistently. The reviewing process has required more time than expected and in some few cases much more than what is considered acceptable. It is essential to get more commitment from the community in order to reduce the time of the reviewing process and support the work of the Associate Editor.

\section{Paper Download Requests via the SciELO System}

While there are several types of indicators, such as the citation index, the impact factor, the h-index, etc., that may have some information about the quality of the paper, a careful analysis of how these indexes are calculated might reveal their limitations. An evidence of this is that indexes rely on databases that are frequently incomplete. There are of course many studies that examine this issue in more depth (See for example the discussions in Calver and Bradley (2009)), our aim here is to present the number of download requests as an indication of the readers interest on papers published in the JBSMSE. 
The following data show that results are more significant for publications of 2006 in comparison with the subsequent years, because the number of downloads depends on how long the paper has been published and available for reading in the online system.

- According to the SciELO database accessed in November $26^{\text {th }}$, 2009, for the year 2006, the ten articles with the largest number of requests through the JBSMSE site are: Rill (2006), Guenther et al. (2006), Siverio \& Figueredo (2006), Grassi Jr. \& Okamoto Jr. (2006), Dhar et al. (2006), Mahapatra \& Patnaik (2006), Tannuri et al. (2006), Alfaro \& Drews (2006), Viana \& Steffen Jr. (2006), Pécora \& Parise (2007). It is noteworthy that Rill (2006) has already more than 3,460 requests and beyond these papers there are other 11 papers with more than 1,030 requests each one.

- According to the SciELO database accessed in November $26^{\text {th }}$, 2009, for the year 2007, the ten articles with the largest number of requests at the JBSMSE site are: Sikdar et al. (2007), Aware et al. (2007), Coelho \& Pinho (2007), Maru \& Tanaka (2007), Barbosa Jr. et al. (2007), Amorim \& Weingaertner (2007), Lucena et al. (2007), Silva et al. (2007), Rodrigues \& Coelho (2007), Paulino \& Kim (2007). It is noteworthy that Sikdar et al. (2007) already have more than 1,850 requests.

- According to the SciELO database accessed in November $26^{\text {th }}$, 2009, for the year 2008, the ten articles with the largest number of requests at the JBSMSE site are: Shirahatti et al. (2008), Silva et al. (2008), Lopes et al. (2008), Cruz et al. (2008), Ibhadode \& Dagwa (2008), Ribeiro et al. (2008), Botelho et al. (2008), Bavastro et al. (2008), Xu et al. (2008), Chemin Filho \& Marcondes (2008). It is noteworthy that Shirahatti et al. (2008) has already 2,370 requests. This number is significantly larger when compared to the number of requests for the papers of the previous year.

- According to the SciELO database accessed in November $26^{\text {th }}$, 2009, (Only the first 2 numbers of Vol. 31 were available) the five papers with the largest number of requests are: Alves et al. (2009), Shetty et al. (2009), Estupiñan \& Ferreira (2009), Pitalude et al. (2009), Costa et al. (2009). It is noteworthy that Ahmed et al. (2009) have already about 519 requests.

\section{Final Comments}

In the past few decades, we have been witnessing a fast increase of academic research in both Science and Engineering in global scale. This is of course accompanied by increases of human resources in the university system and financial support by both governmental agencies and industrial sector. In Brazil, with the recent expansion of the university system and economic growth, we are experiencing the same phenomena.

In this competitive environment, researchers are always under pressure for quantitative indexes as evaluations for promotions are always periodic. It is in this scenario that we must understand the role of the JBSMSE. There is no doubt that the SciELO system is of fundamental importance. The journal reachability would be largely reduced if only the printed version was available. This can be evidenced by the recent increase of paper manuscript submissions from abroad, especially from China and India.

We must be conscious that it is necessary to establish a continuous quality improvement cycle of the JBMSE. Not only the quality translated by the technical content of the paper, but the quality of services that are provided for the whole journal workflow.

\section{Acknowledgements}

Finally, we would like to note that the JBSMSE has already a history of more than 31 years and is consolidated as a high quality scientific journal and of increasing recognition by the international community. While achieving this status, beyond the editorial board, there is a whole team of volunteer collaborators that ensure working conditions for professors and researchers involved in the evaluation and reviewing contents of the JBSMSE, and also others whose work results in the printing version and the online electronic version of the JBSMSE.

On behalf of the community of readers, authors of papers, reviewers and editors of the JBSMSE team, we would like to express special thanks to all these volunteers.

\section{References}

Astarita, G. \& Greco, G., 1968, "Excess Pressure Drop in Laminar Flow Through Sudden Contraction”, Ind. Eng. Chem. Fundam, Vol. 7, No. 1, pp. 27-31.

Alfaro, S.C.A. and Drews, P. (2006) "Intelligent systems for welding process automation”. J. Braz. Soc. Mech. Sci. \& Eng., Vol. 28, No. 1, pp. 25-29.

Alves, J.A.C. et al. (2009) "Application of the Minimum Quantity Lubrication (MQL) technique in the plunge cylindrical grinding operation”. J. Braz. Soc. Mech. Sci. \& Eng., Vol. 31, No. 1, pp. 1-4.

Amorim, F.L. and Weingaertner, W.L. (2007) "The behavior of graphite and copper electrodes on the finish die-sinking electrical discharge machining (EDM) of AISI P20 tool steel”. J. Braz. Soc. Mech. Sci. \& Eng., Vol. 29, No. 4, pp. 366-371.

Awari, G.K. et al. (2007) "A generalized gas-liquid two-phase flow analysis for efficient operation of airlift pump”. J. Braz. Soc. Mech. Sci. \& Eng., Vol. 29, No. 3, pp. 307-312.

Barbosa Jr., J.R. (2007) "Flow boiling of water in a vertical tube at subatmospheric pressures”. J. Braz. Soc. Mech. Sci. \& Eng., Vol. 29, No. 4, pp. 401-409.

Bastos-Netto, D. et al. (1997) "Theoretical formulation for sauter mean diameter of pressure-swirl Atomizers". Journal of Propulsion and Power, Vol. 13, No. 5, pp. 691-696.

Bavastri, C.A. et al. (2008) "Modeling of dynamic rotors with flexible bearings due to the use of viscoelastic materials”. J. Braz. Soc. Mech. Sci. \& Eng., Vol. 30, No. 1, pp. 22-29.

Botelho, E.C. et al. (2008) "Hygrothermal effects evaluation using the iosipescu shear test for glare laminates”. J. Braz. Soc. Mech. Sci. \& Eng., Vol. 30, No. 3, pp. 213-220.

Calver, M.C. and Bradley, J.S. (2009) "Should we use the mean citations per paper to summarise a journal's impact or to rank journals in the same field?", Scientometrics, Vol. 81, No. 3, pp. 611-615.

Caurin, G.A.P. et al. (2001). The influence of the RBF training strategy on the performance of a neural gripper”. Advanced Robotics, Vol. 14, No. 8, pp. 651-742.

Chemin-Filho, R.A. and Marcondes, P.V.P. (2008) "True strain distribution profile on sheet metal using different punch geometries”. $J$. Braz. Soc. Mech. Sci. \& Eng., Vol. 30, No. 1, pp. 1-6.

Costa, E.S. et al. (2009) "Burr produced on the drilling process as a function of tool wear and lubricant-coolant conditions”. J. Braz. Soc. Mech. Sci. \& Eng., Vol. 31, No. 1, pp. 57-63.

Cruz, A.G.B. et al. (2008) "Minimum pressure coefficient criterion applied in axial-flow hydraulic turbines”. J. Braz. Soc. Mech. Sci. \& Eng., Vol. 30, No. 1, pp. 30-38.

Cunha, F.R and Abade, G.C. (2007) "Computer simulation of particle aggregates during sedimentation”. Computer Methods in Applied Mechanics and Engineering, Vol. 196, pp. 4597-4612.

De Marqui Jr., C. et al. (2006) "Identification of flutter parameters for a wing model”. J. Braz. Soc. Mech. Sci. \& Eng., Vol. 28, No. 3, pp. 339-346.

Dhar, N.R. et al. (2006) "Wear behavior of uncoated carbide inserts under dry, wet and cryogenic cooling conditions in turning C-60 steel”. $J$. Braz. Soc. Mech. Sci. \& Eng., Vol. 28, No. 2, pp. 146-152.

Diniz, A.E. and Micaroni, R. (2007) "Influence of the Direction and Flow Rate of the Cutting Fluid on Tool Life in Turning Process of AISI 1045 Steel”. International Journal of Machine Tools \& Manufacture, Vol. 47, p. 247-254, 2007.

Estupiñan, E.A. and Santos, I.F. (2009) "Modelling hermetic compressors using different constraint equations to accommodate multibody dynamics and hydrodynamic lubrication”. J. Braz. Soc. Mech. Sci. \& Eng., Vol. 31, No. 1, pp. 35-46.

Grassi Jr., V. and Okamoto Jr., J. (2006) "Development of an omnidirectional vision system”. J. Braz. Soc. Mech. Sci. \& Eng., Vol. 28, No. 1, pp. 58-68. 
Guenther, R. et al. (2006) "Cascade controlled pneumatic positioning system with LuGre model based friction compensation”. J. Braz. Soc. Mech. Sci. \& Eng., Vol. 28, No. 1, pp. 48-57.

Ibhadode, A.O.A. and Dagwa, I.M. (2008) "Development of asbestosfree friction lining material from palm kernel shell”. J. Braz. Soc. Mech. Sci. \& Eng., Vol. 30, No. 2, pp. 166-173.

Lopes, K.S.S. et al. (2008) "Influence of machining parameters on fatigue endurance limit of AISI 4140 steel”. J. Braz. Soc. Mech. Sci. \& Eng., Vol. 30, No. 1, pp. 77-83.

Lucena, S.E. et al. (2007) "Low-cost PWM speed controller for an electric mini-baja type vehicle”. J. Braz. Soc. Mech. Sci. \& Eng., Vol. 29, No. 1, pp. 21-25.

Mahapatra, S.S. and Patnaik, A. (2006) "Parametric optimization of wire electrical discharge machining (WEDM) process using Taguchi method”. $J$. Braz. Soc. Mech. Sci. \& Eng., Vol. 28, No. 4, pp. 422-429.

Maru, M.M. and Tanaka, D.K. (2007) "Consideration of stribeck diagram parameters in the investigation on wear and friction behavior in lubricated sliding”. J. Braz. Soc. Mech. Sci. \& Eng., Vol. 29, No. 1, pp. 55-62.

Maruyama, N. and Souza, E.C. (2007) "Inteligent UUV's: some issues on ROV dynamic positioning”. IEEE Transactions on Aerospace and Electronic Systems, Vol. 43, pp. 214-226.

Morooka, C.K. et al. (2001) "Development of intelligent systems for well drilling and petroleum production”. Journal of Petroleum Science \& Engineering, Vol. 32, pp. 191-199.

Naccache, M.F. et al. (2007). "Flow of viscoplastic liquids through axisymmetric expansions-contractions”. Journal of Non-Newtonian Fluid Mechanics (Print), Vol. 142, pp. 207-217.

Paulino, G.H. and Kim, J.-H. (2007) "The weak patch test for nonhomogeneous materials modeled with graded finite elements”. J. Braz. Soc. Mech. Sci. \& Eng., Vol. 29, No. 1, pp. 63-81.

Pécora, A.A.B. and Parise, M.R. (2006) "Heat transfer coefficient in a shallow fluidized bed heat exchanger with a continuous flow of solid particles”. J. Braz. Soc. Mech. Sci. \& Eng., Vol. 28, No. 3, pp. 253-258.

Pintaude, G. et al. (2009) "Plastic deformation analysis of low-carbon steel due to metal hole punching using coated and uncoated tools”. J. Braz. Soc. Mech. Sci. \& Eng., Vol. 31, No. 1, pp. 52-56.

Rade, D.A. et al. (2006) "Identification of welding residual stresses in rectangular plates using vibration responses”. Inverse Problems in Engineering, Vol. 14, No. 3, pp. 313-331.

Ribeiro, J.C.L. et al. (2008) "Eurocode structural fire design and its application for composite circular hollow section columns”. J. Braz. Soc. Mech. Sci. \& Eng., Vol. 30, No. 1, pp. 39-46.
Rill, G. (2006) “Vehicle modeling by subsystems'. J. Braz. Soc. Mech. Sci. \& Eng., Vol.28, No.4, pp.430-442.

Rodrigues, A.R. and Coelho, R.T. (2007) "Influence of the tool edge geometry on specific cutting energy at high-speed cutting”. J. Braz. Soc. Mech. Sci. \& Eng., Vol. 29, No. 3, pp. 279-283.

Savi, M.A. et al. (2009). "Lyapunov exponents estimation for hysteretic systems”. International Journal of Solids and Structures, Vol. 46, pp. 1269-1598.

Shetty, R. et al. (2009) "Taguchi's technique in machining of metal matrix composites”. J. Braz. Soc. Mech. Sci. \& Eng., Vol. 31, No. 1, pp. 12-20.

Shirahatti, A. et al. (2008) "Optimal design of passenger car suspension for ride and road holding”. J. Braz. Soc. Mech. Sci. \& Eng., Vol. 30, No. 1, pp. 66-76.

Shirahatti, A. et al. (2008) "Optimal design of passenger car suspension for ride and road holding”. J. Braz. Soc. Mech. Sci. \& Eng., Vol. 30, No. 1, pp. 66-76.

Sikdar, S. et al. (2007) "Analysis of roll stack deflection in a hot strip mill”. J. Braz. Soc. Mech. Sci. \& Eng., Vol. 29, No. 3, pp. 284-289.

Silva, A.L.F.L. et al. (2007) "Numerical simulation of two-dimensional complex flows around bluff bodies using the immersed boundary method". J. Braz. Soc. Mech. Sci. \& Eng., Vol. 29, No. 4, p. 379-387.

Silva, J.G.S. et al. (2008) "Vibration analysis of orthotropic composite floors for human rhythmic activities”. J. Braz. Soc. Mech. Sci. \& Eng., Vol. 30, No. 1, pp. 56-65.

Silvério, R.J.R. and Figueredo, J.R. (2006) "Steady state simulation of the operation of an evaporative cooled water-ammonia absorption scale ice maker with experimental basis”. J. Braz. Soc. Mech. Sci. \& Eng., Vol. 28, No. 4, pp. 413-421.

Tannuri, E.A. et al. (2006) “Adaptive techniques applied to offshore dynamic positioning systems”. J. Braz. Soc. Mech. Sci. \& Eng., Vol. 28, No. 3, pp. 323-330.

Viana, F.A.C. and Steffen Jr., V. (2006) "Multimodal vibration damping through piezoelectric patches and optimal resonant shunt circuits". J. Braz. Soc. Mech. Sci. \& Eng., Vol. 28, No. 3, pp. 293-310.

$\mathrm{Xu}, \mathrm{Z}$. et al. (2008) "Trajectory planning of jumping over obstacles for hopping robot”. J. Braz. Soc. Mech. Sci. \& Eng., Vol. 30, No. 4, pp. 327-334.

Willinsky, J (2005). "Open Journal Systems: an example of open source software for journal management and publishing”. Library Hi-Tech, Vol. 23, No. 4, pp. 504-519.

Zouain, N. et al. (2006). "Using enclosing ellipsoids in multiaxial fatigue strength criteria”. European Journal of Mechanics. A, Solids, Vol. 25, No. 1, pp. 51-71. 